\title{
Tumor maligno de la vaina del nervio periférico (MPNST) glandular de la órbita: primera descripción de la literatura de localización orbitaria en un paciente con
} neurofibromatosis tipo 1

\author{
A.E. Romero-Rojas*; J.A. Díaz-Pérez*,** y A. Lozano-Castillo*
}

Grupo de Patología del Instituto Nacional de Cancerología*. Bogotá. Colombia. Fundación para el Avance de la Anatomía Patológica, Citología y Clínica Molecular FAPCIM**. Universidad de Santander UDES. Bucaramanga. Colombia.

Resumen

Introducción. El tumor maligno de la vaina del nervio periférico (MPNST, por sus siglas en ingles Malignant Peripheral Sheath Tumor), es una neoplasia maligna originada en las células de Schwann de la vaina de revestimiento de los nervios periféricos. Esta neoplasia puede presentar componentes heterólogos benignos o malignos, con diferenciación divergente, como la diferenciación glandular.

Objetivo. Describir el primer caso en la literatura de MPNST glandular maligno localizado a nivel orbitario y realizar una revisión sobre esta neoplasia.

Caso clínico. Niño de 9 años de edad, con diagnostico de NF1, quien presentó exoftalmos ocular, dolor retro-ocular, cefalea, asimetría facial y descenso del globo ocular derecho de 1 año de evolución; a quien se documento masa sólida orbitaria, delimitada, lobulada, que se proyecta al parénquima cerebral frontal y temporal en los estudios de tomografía computarizada y resonancia magnética. La lesión se abordó en forma fronto-orbito-cigomática con resección completa de la misma. Posteriormente, se hizo una plastia dural en base de cráneo y reconstrucción con malla de titanio. Actualmente el paciente se encuentra asintomático después de 6 meses de tratamiento. En el estudio anatomopatológico se observó una neoplasia maligna bifásica, reactiva en los elementos mesenquimales para S100, PGP 9.5, neurofilamentos y vimentina. El componente glandular fue positivo para AE1/AE3, EMA, CEA y focalmente para CD57. Se observó además reactividad para cromogranina, sinaptofisina, serotonina y somatostatina. Se realizo el diagnostico de MPNST glandular de la órbita.

Conclusión. Se describe el primer caso de MPNST glandular localizado en la órbita, el cual se presentó en un niño con NF1. Esta neoplasia extremadamente infrecuente debe ser tenida en cuenta en el estudio de lesiones bifásicas malignas, ya que su diagnostico es de

Recibido: 6-05-09. Aceptado: 20-09-09 peculiar importancia debido al pésimo pronóstico de los pacientes afectados.

PALABRAS CLAVE: Tumor maligno de la vaina del nervio periférico. Glándula. Órbita. Caso clínico. Revisión de la literatura. (Fuente: DeCS).

Malignant peripheric nerve sheath tumor of the orbit: First description of orbital location in a patient with NF1.

Summary

Introduction. The malignant peripheric nerve sheath tumor (MPNST), is a malignant neoplastic lesion originated in Schwann cells of the lining sheath of peripheral nerves. This neoplasia may appear with benign or malignant heterologous components, with divergent differentiation, as the glandular one.

Aim. To describe for the first time in the literature, a case of a glandular MPNST, located at the orbit and to revise the literature on this tumoral lesion.

Clinical case. Nine year old male, with a base diagnosis of NF1, who had exophthalmos, retro-ocular pain, headache, facial asymmetry and descent of the right eyeball, that started 1 year earlier. This patient showed in the Computed Tomography an Magnetic Resonance, a well delimited, lobulated, solid mass at the eyeball, which extended to the fontal and temporal brain parenchyma. A right Fronto-temporal craniotomy was made with fronto -orbital-zygomatic resection of the tumoral lesion. Later, a dural plasty and reconstruction with titanium mesh was made at the skull base. At present, the patient is asymptomatic after 4 months of follow up. A malignant biphasic neoplastic lesion was observed, reactive in the mesenchymal elements S100, PGP 9.5, neurofilaments and vimentin. The glandular component was positive for AE1/AE3, EMA, CEA and focally for CD57. There was also reactivity to cromogranin, synaptophysin, serotonin and somatostatin. The diagnosis of Glandular MPNST was made. 
Conclusion. For the first time in the literature a case of Glandular MPNST located at the orbit, which occurred in child with NF1, is described. This extremely uncommon neoplasia must be taken into account, in the study of biphasic malignant lesions, as its diagnosis is of great importance because of the bad prognosis of the affected patients.

KEYS WORDS: Malignant peripheral nerve sheath tumor. Gland. Epithelioid. Orbit. Case report. Review (Source: $\mathrm{MeSH})$.

\section{Introducción}

El tumor maligno de la vaina del nervio periférico (MPNST, por sus siglas en ingles Malignant Peripheral Sheath Tumor), también denominado schwannoma maligno, neurofibrosarcoma, neurilemoma maligno y sarcoma neurogénico, es una lesión neoplásica maligna originada en las células de Schwann de la vaina del revestimiento de los nervios periféricos ${ }^{27,33}$. Se localiza con mayor frecuencia en las partes profundas de la cabeza, cuello, columna y extremidades inferiores ${ }^{18}$. Esta neoplasia, es la responsable de alrededor del 5\% de los sarcomas de tejidos blandos, con una incidencia de 1 caso por millón de personas-año ${ }^{33}$. Se ha asociado a diferentes factores, siendo el más implicado (hasta en el $50 \%$ de los casos), la presencia de neurofibromatosis 1 (NF1) $)^{10,14}$; enfermedad en la que se originan múltiples neurofibromas, algunos con capacidad de transformación a un MPNST, entre los que se destacan los neurofibromas plexiformes los cuales presentan un mayor riesgo de progresión hacia la malignidad ${ }^{1,14,32}$. El desarrollo de los MPNST también se ha asociado a la exposición a radiación, presentándose desde 4 a 40 años después de la misma, y a la presencia de ganglioneuromas ${ }^{10}$. Sin embargo, en el $50 \%$ de los casos no se encuentran características asociadas ${ }^{10}$. Los MPNST tienen diferentes particularidades, entre las que se destacan su capacidad de recurrencia locorregional y de metástasis a distancia ${ }^{3}$; además de la inusual diferenciación (20\%) a componentes heterólogos benignos o malignos ${ }^{11}$, con diferenciación divergente, como la glandular, rabdomiosarcomatosa, osteosarcomatosa, condrosarcomatosa y melánica $^{13,19}$. La diferenciación glandular es extremadamente rara, menos del 1\% de los MPNST la desarrollan, ésta puede ser benigna o maligna, al igual que la diferenciación mesenquimal ${ }^{19,30}$. Las variedades con elementos malignos glandulares han sido referidas como MPNST glandular o schwannoma maligno epiteloide glandular. Este tipo de diferenciación fue descrita inicialmente por Garre en 1892, al informar 5 casos de este tipo particular de tumor ${ }^{9}$, posteriormente se han informado pocos casos, alrededor de 50 , 8 de ellos malignos, ninguno localizado en la órbita, los cuales se asocian con un peor pronóstico que los MPNST clásicos $^{13,19,30}$. Por lo cual, se describirá el primer caso en la literatura de MPNST glandular maligno localizado a nivel orbitario en un niño con NF1, y realizará una revisión sobre esta neoplasia.

\section{Caso clínico}

Paciente de sexo masculino de 9 años de edad, con diagnostico de NF1, quien presentó exoftalmos ocular, dolor retro-ocular, cefalea, asimetría facial y descenso del globo ocular derecho de 1 año de evolución. La exploración neurológica demostró adecuados movimientos oculares, campos oculares normales, agudeza visual normal, córnea clara, múltiples nódulos de Lisch de predominio palpebral inferior en ojo derecho, cristalino claro y pupila isocórica reactiva; al fondo de ojo se evidencio papila rosada de bordes definidos, mácula sana y periferia sin alteraciones; además de manchas café con leche generalizadas e hiperpigmentación axilar. La TC de cráneo evidencio masa sólida orbitaria, delimitada, lobulada, que se proyecta al parénquima cerebral frontal y temporal, compatible con neurofibroma plexiforme, y ausencia del ala mayor derecha temporal. La resonancia magnética (RM) de órbitas, mostró ausencia del ala mayor del esfenoides derecho, con herniación del lóbulo temporal hacia la órbita, con exoftalmos, microftalmos y rechazo del nervio óptico; en la órbita se identifico extensa masa isointensa a la sustancia gris cerebral en todas las secuencias T2, la cual realzó con la instalación de medio de contraste, de contornos mal definidos y extensión a la fosa craneal media; no se observaron colecciones compresivas, ni signos de hidrocefalia activa (Figura 1). Con lo anterior, se consideró la presencia de neurofibroma plexiforme orbitario derecho acompañado de microftalmos. Fue llevado a craneotomía fronto-temporal derecha, en la cual se halló extensa masa orbitaria que se desplazaba a la región etmoidal y fosa anterior de aspecto sarcomatoso, se realizo resección fronto-orbito-cigomática, disecando y extrayendo la masa tumoral de $8 \mathrm{~cm}$ de diámetro mayor en forma fragmentada, por múltiples adherencias con el nervio óptico. Posteriormente, se hizo una plastia dural a nivel de base de cráneo, con reconstrucción del piso de la órbita media y anterior, con malla de titanio. Actualmente el paciente se encuentra asintomático después de 6 meses de seguimiento.

\section{Estudio anatomopatológico}

Macroscópicamente se recibieron múltiples fragmentos de tejido de color blanco amarillento, firmes. Microscópicamente se evidencio el aspecto morfológico de un neurofibroma de patrón plexiforme (Figura 2A). La gran mayoría del tumor estaba compuesto por células fusiformes, 

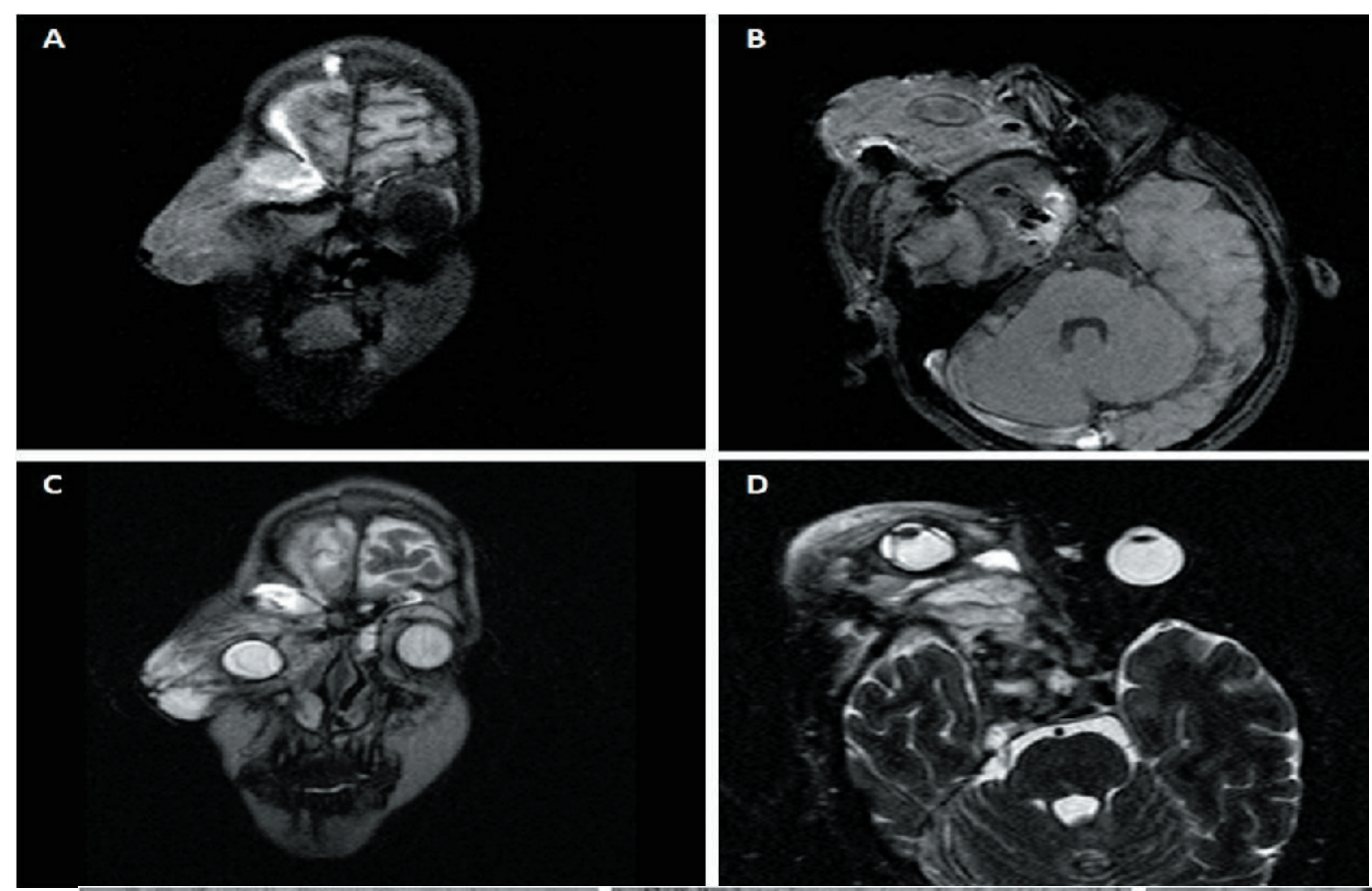

Figura 1. En la figura se observan imágenes de RMN en los que se detalla la ausencia del ala mayor del esfenoides derecho, y extensa masa, que producía exoftalmos, microftalmos y rechazo del nervio óptico en la órbita derecha (A y B), la cual fue isointensa a la sustancia gris cerebral en todas las secuencias $T 2$, realzando con el medio de contraste (Cy D).
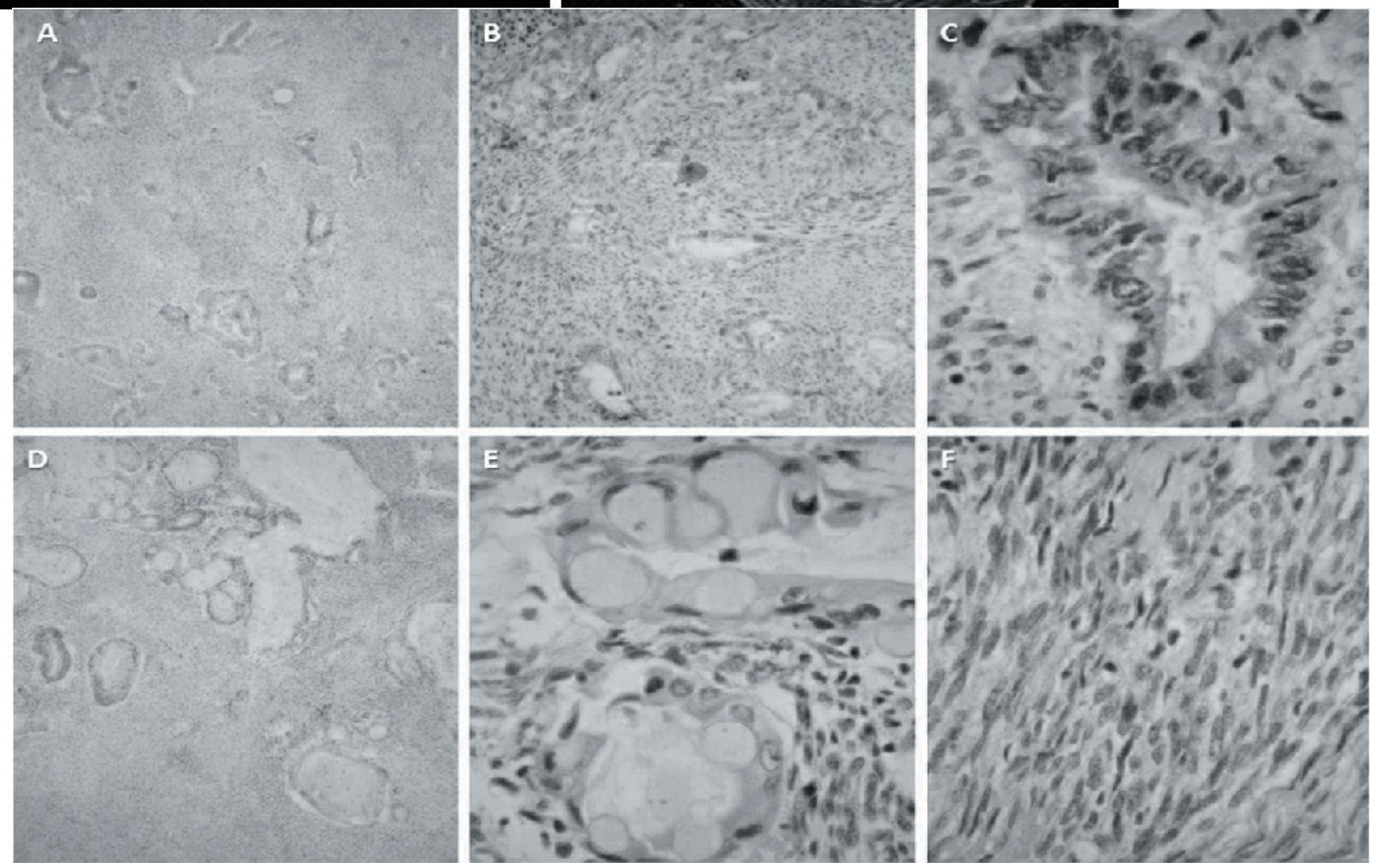

Figura 2. En el estudio histopatologico se observó una lesión tumoral con características de neurofibroma de patrón plexiforme, con dos componentes $(A$ y B). El primero de ellos estaba conformado por estructuras glandulares malignas tapizadas por células cilíndricas con citoplasma vacuolado de aspecto secretor intestinal con marcada atipia y estratificación nuclear con frecuentes figuras mitóticas atipicas (C, D y E). El segundo componente estaba compuesto por gran cantidad de células fusiformes, elongadas, con áreas de atipia nuclear, con marcado pleomorfismo e hipercromatismo asociado a la presencia de numerosas figuras mitóticas incluyendo formas atípicas (F). No se identificó la presencia de células mioepiteliales en los elementos glandulares. 
Tabla 1

Características del panel de inmunohistoquímica empleado en el estudio del caso

\begin{tabular}{|l|l|l|l|}
\hline \multicolumn{1}{|c|}{ Marcador } & Clon & Dilución & Casa Comercial \\
\hline Queratinas & AE1AE3 & $1: 100$ & DAKO \\
\hline BCL2 & Bcl2/100/D5 & $1: 50$ & Novocastra \\
\hline Cromogranina & DAK-A3 & $1: 100$ & Dako Cytomation \\
\hline EMA & E29 & $1: 50$ & Dako Cytomation \\
\hline CD57 (Leu-7) & NK1 & $1: 200$ & Neomarkers \\
\hline CD99 & HO36-11 & $1: 100$ & Neomarkers \\
\hline Ki67 & MM1 & $1: 200$ & Novocastra \\
\hline S100 & 4 C4.9 & $1: 50$ & Neomarkers \\
\hline Vimentina & V-9 & $1: 200$ & BioGenex \\
\hline Sinaptofisina & SNP88 & $1: 100$ & BioGenex \\
\hline Serotonina & 5 HT-H209 & $1: 40$ & Deomarkers \\
\hline Neurofilamentos & ZF11 & $1: 50$ & Dako Cytomation \\
\hline Somatostatina & I-14 & $1: 200$ & Dako Cytomation \\
\hline P63 & 4 A4 & $1: 25$ & Novocastra \\
\hline CD10 & $56 C 6$ & $1: 100$ & BioGenex \\
\hline Actina músculo Liso (A/ML) & 1 A4 & $1: 300$ & Dako Cytomation \\
\hline PGP9.5 & & $1: 100$ & \\
\hline
\end{tabular}

Tabla 2

Estrategia de búsqueda de la literatura, se utilizan descriptores MeSH para la base de datos MedLine, para las demás bases de datos la búsqueda se realizo en forma análoga.

\begin{tabular}{|l|l|c|}
\hline Paso & Descriptor (Palabra Clave) & Numero de Citaciones \\
\hline 1 & Malignant Peripheral Sheath Tumor & 1.071 \\
\hline 2 & Glandular & 22.309 \\
\hline 3 & Orbit & 8 \\
\hline 4 & 1 AND 2 & 0 \\
\hline 5 & 1 AND 2 AND 3 \\
\hline
\end{tabular}

elongadas con núcleos apincelados, con áreas de atipia nuclear en un espectro desde leve a grave (Figura $2 \mathrm{~F}$ ), con marcado pleomorfismo e hipercromatismo asociado a la presencia de numerosas figuras mitóticas incluyendo formas atípicas. También se identificaron componentes glandulares malignos (Figura 2B) tapizados por células cilíndricas con citoplasma vacuolado de aspecto secretor intestinal (Figura $2 \mathrm{C}, 2 \mathrm{D}$ y $2 \mathrm{E}$ ) con marcada atipia y estratificación nuclear con frecuentes figuras mitóticas atípicas. No se identificó la presencia de células mioepiteliales en los elementos 

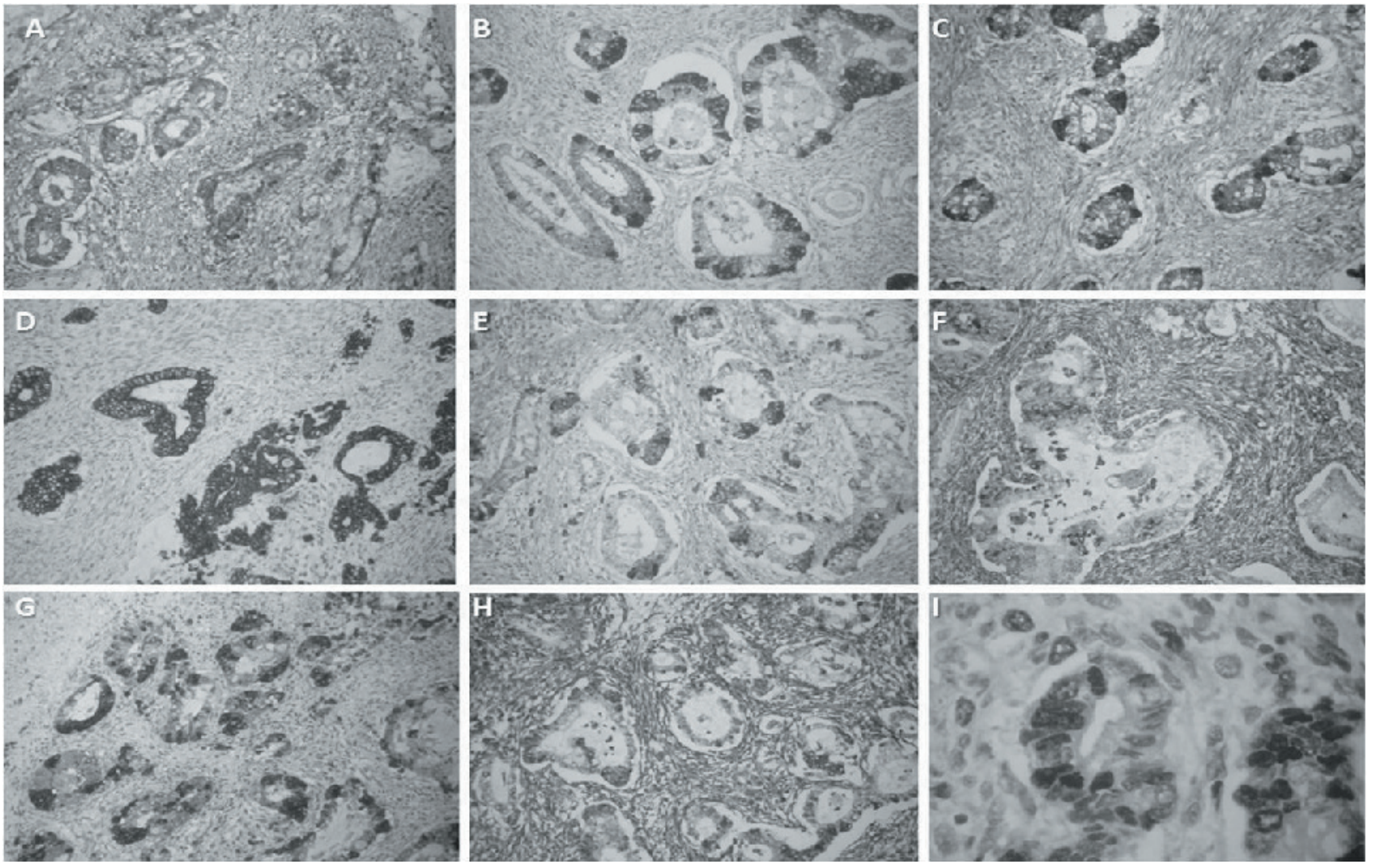

Figura 3. Se observa inmunorreactividad en los elementos mesenquimales para PGP 9.5 (A), neurofilamentos $(F)$ y vimentina (H), y negatividad para AE1AE3 (D). El componente glandular fue fuertemente positivo para AE1/AE3 (D), y focalmente para CD57 (E). El indice de proliferación de celular de Ki67 fue elevado (más del 40\%) tanto en los elementos mesenquimales como epiteliales (I). La cromogranina (B), sinaptofisina $(G)$ y somatostatina $(C)$ evidenciaron fuerte positividad.

glandulares. Morfológicamente no se reconoció diferenciación o componente neuroendocrino asociado. Las características de los estudios de inmunohistoquímica realizados se detallan en la Tabla 1. Los elementos mesenquimales fueron fuertemente positivos para S100, PGP 9.5 (Figura $3 \mathrm{~A}$ ), neurofilamentos (Figura $3 \mathrm{~F}$ ) y vimentina (Figura $3 \mathrm{H}$ ), con focal reactividad para CD99 pero negativos para CEA, AE1AE3 (Figura 3D), EMA y Bcl2. El componente glandular fue fuertemente positivo para queratinas (Figura 3D), EMA, CEA y focalmente para CD57 (Figura 3E). El índice de proliferación de celular Ki67 fue elevado (más del 40\%) tanto en los elementos mesenquimales como epiteliales (Figura 3I). Se observo negatividad para p63, AML y CD10 en las glándulas, confirmando la ausencia de células mioepiteliales. Los marcadores neuroendocrinos para cromogranina (Figura 3B), sinaptofisina (Figura 3G), serotonina y somatostatina (Figura $3 \mathrm{C}$ ) evidenciaron fuerte positividad. Con lo anterior se realizo el diagnostico de MPNST glandular de la órbita. El neurofibroma plexiforme asociado dentro del tumor fue reactivo únicamente para S100 y vimentina.

\section{Estrategia de búsqueda de la literatura}

Se realizó una búsqueda estructurada de la literatura, con base en la metodología Cochrane, en las bases de datos Medline, Imbiomed y Scielo, utilizando las palabras clave "Malignant Peripheral Sheath Tumor", "glandular", y "orbit" desde el año 1990 a Marzo de 2009, limitando la búsqueda a artículos originales escritos en idioma inglés y español, la metodología empleada se muestra en la Tabla 2. De los artículos encontrados, se seleccionaron los considerados relevantes por los autores.

\section{Discusión}

Los MPNST desarrollan en forma relativamente común, $20 \%$ de los casos, cambios metaplásicos epiteliales y mesenquimales, de características benignas o malignas ${ }^{11}$. Los elementos mesenquimales son los más comúnmente encontrados, y entre ellos el cartílago, músculo y el hueso, son los observados con mayor frecuencia, por lo anterior, los elementos malignos generalmente vistos 
son de sarcomas de alto grado de tipo rabdomiosarcoma, osteosarcoma, condrosarcoma y angiosarcoma. También se han identificado otros elementos heterólogos de origen mesenquimal y epitelial, estos últimos pueden observarse como células escamosas, formaciones glandulares, células indiferenciadas y células neuroendocrinas ${ }^{19,30}$. La diferenciación glandular de los MPNST es un fenómeno muy inusual, en nuestra búsqueda encontramos 8 casos, 5 de ellos totalmente documentados. Esta diferenciación glandular se ha asociado con la enfermedad de von Recklinghausen, gracias a los trabajos de Woodruff y Christen$\operatorname{sen}^{5,30}$.

La génesis de estos tumores con diferenciación divergente es controvertida, para explicarla se han formulado dos teorías. La primera de ellas se basa en el posible origen de los MPNST a partir de la migración anormal de células madre de la cresta neural, que tendrían la capacidad de diferenciarse a elementos neuroectodérmicos como melanocitos, células ganglionares, células de Schwann y células multipotenciales, estas últimas serían las responsables del desarrollo de elementos heterólogos, como las formaciones glandulares ${ }^{5}$. Lo anterior está sustentado en que cuando se desarrollan elementos epiteliales, estos suelen ser glandulares con características intestinales neuroendocrinas productoras de péptidos y aminas, como serotonina, somatostatina y gastrina ${ }^{5,26}$. La segunda teoría que puede explicar el origen de las glándulas de los MPNST con diferenciación glandular, se basa en la presencia de elementos ependimales heterotópicos, la cual no ha tenido gran acogida por la ausencia de pruebas moleculares y ultraestructurales que la soporten ${ }^{7,8,15,22}$.

Clínicamente los MPNST con diferenciación glandular poseen un comportamiento más agresivo y un peor desenlace $^{19}$, aunque se han descrito casos de comportamiento favorable ${ }^{2}$. Estos suelen ser sintomáticos con un menor tamaño, presentan un crecimiento más rápido $\mathrm{y}$ pueden invadir estructuras adyacentes ${ }^{3,19}$, además la sobrevida libre de enfermedad luego de la resección es menor y tienen una mayor tasa de metástasis ${ }^{3}$. La edad media de presentación de los MPNST con diferenciación glandular es de 28 años, muy similar a los MPNST puros ${ }^{19}$. En estas lesiones no se han documentado diferencias por género.

En el estudio patológico de la pieza quirúrgica, se caracterizan macroscópicamente por ser grandes masas regulares localizadas adyacentes a un nervio, de consistencia firme, que se disponen en forma fusiforme y que presentan múltiples áreas de degeneración quística y mixoide ${ }^{4,27,33}$. En el estudio histopatológico, se reconocen elementos mesenquimales malignos, compuestos por gran cantidad de células pleomorfas dispuestas en fascículos, conformando ocasionales empalizadas y espacios pseudovasculares ${ }^{24,33}$. Las células que conforman esta lesión tienen la particularidad de presentar mitosis frecuentes y ocasio- nal morfología bizarra. Además, se encuentran células tumorales distribuidas en forma perivascular. Otros hallazgos relevantes son la presencia de necrosis geográfica y de metaplasia mesenquimal, que puede ser condroide, ósea o muscular ${ }^{27,24}$. Los componentes epiteliales son comúnmente benignos, cuando se presentan componentes epiteliales malignos, se observan frecuentes figuras mitóticas, prominentes nucleolos y marcado pleomorfismo, como en nuestro caso $^{19}$. A la presencia de estos elementos epiteliales malignos se ha asociado la peor sobrevida de los pacientes con MPNST diferenciación glandular. Para el diagnostico de los MPNST con diferenciación glandular Woodruff and Christensen formularon una serie de $\operatorname{criterios}^{5,30}$, los cuales son:

1. El tumor se encuentra claramente asociada con un nervio y posee evidencia morfológica de componentes neurofibromatosos.

2. Las formaciones glandulares tienen ausencia de células mioepiteliales.

3. Las células glandulares son reactivas para marcadores epiteliales, como queratinas y EMA, lo cual excluye la posibilidad de una diferenciación pseudoglandular.

4. Las células glandulares presentan frecuentemente marcadores neuroendocrinos, como cromogranina, somatostatina, Leu-7 (CD57), y calcitonina.

En el estudio inmunohistoquímico las células fusiformes son fuertemente positivas para vimentina y S-10034; observándose también la expresión de otros marcadores como CD99 en frecuencias variables que oscilan en diferentes series desde un 10 al 86\% de los casos, CD 57 en el 55\%, colágeno tipo IV, producto de la proteína genética 9,5 (PGP 9.5) y P5327,19. Los elementos glandulares epiteliales son reactivos para EMA, citoqueratinas (CAM 5.2, AE1/ AE3, 8,19 y 20), y antígeno carcinoembrinario (CEA); reconociendo también marcadores neuroendocrinos ${ }^{12,16,21}$.

El diagnóstico diferencial de los MPNST con diferenciación glandular, se realiza con el sarcoma sinovial bifásico, teratomas, y con las metástasis de adenocarcinoma a Schwannomas benignos, además por la localización de este caso se descartó también la presencia de un meningioma. La distinción entre estos tumores se logra gracias a estudios de inmunohistoquímica que identifiquen componentes glandulares positivos para marcadores epiteliales como células caliciformes, que expresan CEA y marcadores neuroendocrinos que favorecen el diagnostico de MPNST glandular ${ }^{12,16,20,23,31}$. También ocasionalmente se pueden observar estructuras de los anexos cutáneos atrapadas en los TMVNP lo que constituiría una pseudo-diferenciación, la cual debe tenerse en cuenta en el diagnostico apropiado de estas neoplasias ${ }^{8}$. Los MPNST pueden diferenciarse de una forma sencilla con los sarcomas sinoviales bifásicos (SSB) dado que la reactividad de los marcadores epiteliales se expresan únicamente en los elementos epiteliales del 
MPNST, mientras que en los SSB hay positividad tanto en los componentes epiteliales como mesenquimales. Un diagnóstico que en ocasiones genera dificultad son los teratomas con elementos malignos epiteliales y mesenquimales, sin embargo, esta combinación constituye un evento infrecuente y la inmunohistoquímica ayuda en su diferenciación, sobre todo al evaluar los componentes mesenquimales del teratoma ya que de forma muy ocasional los elementos estromales evidencian la diferenciación neural que sí muestran de forma clara los MPNST.

Otras variantes inusuales de esta lesión son la variante epiteloide y el tumor maligno de Tritón. En la variante epiteloide, que corresponde al 5\% de los MPNST, se observa una lesión neoplásica maligna de origen mesenquimal compuesta por células epiteloides con un citoplasma acidofílico; esta variante es la más comúnmente vista en transformación maligna de los neurofibromas no asociados con neurofibromatosis; a nivel de inmunohistoquímica, esta lesión presenta reacción a HMB45, por lo que se debe plantear el diagnóstico diferencial con un melanoma desmoplástico, para lo que es de gran ayuda la positividad inmunofenotípica para el producto del gen 9.5 y la negatividad para proteína $\mathrm{S} 100^{16}$. Por otro lado, el tumor maligno de Tritón, o MPNST con diferenciación rabdomiosarcomatosa ${ }^{25,28}$, descrito inicialmente por Masson en 1932, denominado así debido a que puede ser inducido al contacto por las salamandras tipo Tritón; trabajo realizado por Locatelly en 1925, se establece utilizando los criterios que Woodruff formulo en $1972^{29}$ para su diagnóstico, que son: 1. Tumor originado en el nervio periférico, en un ganglioneuroma, o en un paciente con NF1. 2. Tumor con características de crecimiento propias de las células de Schwann. 3. Que existan rabdomioblastos presentes en el tumor.

El tratamiento de esta enfermedad es netamente quirúrgico planteándose como meta la resección con márgenes histopatológicos libres entre 3 y $5 \mathrm{~cm}$, es de gran importancia resaltar que en ocasiones puede requerirse resecciones en bloque que pueden llevar a alteraciones funcionales ${ }^{6}$. En neoplasias irresecables o metastásicos se ha planteado la opción de la quimioterapia presentando una respuesta más favorable en aquellos pacientes que no cursan con una neurofibromatosis tipo 1; el régimen terapéutico mas estudiado es el de ifosfamida + doxurrubicina; la radioterapia aunque no ha demostrado mejorar la sobrevida puede llevar al control local del tumor en pacientes con la enfermedad irresecable localmente avanzada ${ }^{17}$.

En conclusión, se describe el primer caso de MPNST glandular localizado en la órbita, el cual se presento en un niño con NF1. Esta neoplasia extremadamente infrecuente debe ser tenida en cuenta en el estudio de lesiones bifásicas malignas, ya que su diagnostico es de peculiar importancia debido al pésimo pronostico de los pacientes afectados.

\section{Bibliografía}

1. Agesen, T.H., Florenes, V.A., Molenaar, W.M., et al.: Expression patterns of cell cycle components in sporadic and neurofibromatosis type 1-related malignant peripheral nerve sheath tumors. J Neuropathol Exp Neurol. 2005; 64: 74-81.

2. Allegranza, A., Ferraresi, S., Luccarelli, G.: Malignant glandular schwannoma: a case with favourable prognosis. Histopathology. 1988; 12: 549-52. No abstract available.

3. Anghileri, M., Miceli, R., Fiore, M., et al.: Malignant peripheral nerve sheath tumors: Prognostic factors and survival in a series of patients treated at a single institution. Cancer 2006; 107: 1065-1074.

4. Carli, M., Ferrari, A., Mattke, A., et al.: Pediatric malignant peripheral nerve sheath tumor: The Italian and German soft tissue sarcoma cooperative group. J Clin Oncol 2005; 23: 8422- 8430.

5. Christensen, W.N., Strong, E.W., Bains, M.S., Woodruff, J.M.: Neuroendocrine differentiation in the glandular peripheral nerve sheath tumor. Pathologic distinction from the biphasic synovial sarcoma with glands. Am J Surg Pathol. 1988; 12: 417-426.

6. Coindre, J.M., Terrier, P., Bui, N.B., et al.: Prognostic factors in adult patients with locally controlled soft tissue sarcoma. A study of 546 patients from the French Federation of Cancer Centers Sarcoma Group. J Clin Oncol. 1996; 14 : 869-877.

7. Deschryever, K., Santa Cruz, D.J.: So-called glandular schwannoma: ependymal differentiation in a case. Ultrastruct Pathol. 1984; 6: 167-175.

8. Elston, D.M., Bergfeld, W.F., Biscotti, C.V., McMahon, J.T.: Schwannoma with sweat duct differentiation. J Cutan Pathol 1993; 20: 254-258.

9. Garre, C.: Weber sekundai Maligne Neurome Beitrz Klin. Chirurg 1892; 9: 465-495.

10. Grobmyer, S.R., Reith, J.D., Shahlaee, A., Bush, C.H., Hochwald, S.N.: Malignant Peripheral Nerve Sheath Tumor: molecular pathogenesis and current management considerations. J Surg Oncol. 2008; 97: 340-349.

11. Huang, L., Espinoza, C., Welsh, R.: Malignant peripheral nerve sheath tumor with divergent differentiation. Arch Pathol Lab Med. 2003; 127: e147-50.

12. Karabela-Bouropoulou, V., Antoniou, D., LiapiAvgeri, G.: Malignant peripheral nerve sheath tumor with glandular differentiation. Report of a case with emphasis to the usefulness of immunohistochemistry in the differential diagnosis. Arch Anat Cytol Pathol. 1996; 44: 263-268.

13. Karpuz, V., Letovanec, N., Von Hochstetter, A., Joris, F.: Malignant peripheral nerve sheath tumor with rhabdomyoblastic differentiation and glandular component. Ann Pathol. 2000; 20: 62-65

14. Korf, B.: Malignancy in Neurofibromatosis type 1. The Oncologist 2000; 5: 477-485 
15. Lanford, J.A., Cohn, L.: Ependymal neoplasm of the median nerve with case report. South Med J. 1987; 20: 273-278.

16. Lodding, P., Kindblom, L.G., Angervall, L.: Epithelioid malignant schwannoma: a study of 14 cases. Virchows Arch A Pathol Anat Histopathol 1986; 409: 433-451.

17. Masui, F., Yokoyama, R., Soshi, S., et al.: A malignant peripheral nerve sheath tumor responding to chemotherapy. J Bone Joint Surg Br 2004; 86: 113-115.

18. Minovi, A., Basten, O., Hunter, B., Draf, W., Bockmühl, U.: Malignant peripheral nerve sheath tumors of the head and neck: management of 10 cases and literature review. Head Neck. 2007; 29: 439-445.

19. Nagasaka, T., Lai, R., Sone, M., Nakashima, T., Nakashima, N.: Glandular malignant peripheral nerve sheath tumor: an unusual case showing histologically malignant glands. Arch Pathol Lab Med. 2000; 124: 1364-1368.

20. O'Connell, J.X., Browne, W.L., Gropper, P.T., Berean, K.W.: Intraneural biphasic synovial sarcoma: an alternative "glandular" tumor of peripheral nerve. Mod Pathol. 1996; 9: 738-741.

21. Olsen, S.H., Thomas, D.G., Lucas, D.R.: Cluster analysis of immunohistochemical profiles in synovial sarcoma, malignant peripheral nerve sheath tumor, and Ewing sarcoma. Mod Pathol. 2006; 19: 659-668.

22. Papanicolaou, S.J., Eversole, L.R.: Glandular structures in neural sheath neoplasms. Oral Surg Oral Med Oral Pathol 1982; 53: 69-72.

23. Rose, D.S., Wilkins, M.J., Birch, R., Evans, D.J.: Malignant peripheral nerve sheath tumour with rhabdomyoblastic and glandular differentiation: immunohistochemical features. Histopathology. 1992; 21: 287-290.

24. Scheithauer, B.W., Woodruff, J.M., Erlandson, R.A.: Primary malignant tumors of the peripheral nerve. In: Tumors of the Peripheral Nervous System. Washington, DC: Armed Forces Institute of Pathology; 2000: 336-340. Atlas of Tumor Pathology; $3^{\text {rd }}$ series, fascicle 11.

25. Stasik, C.J., Tawfik, O.: Malignant peripheral nerve sheath tumor with rhabdomyosarcomatous differentiation (malignant triton tumor). Arch Pathol Lab Med. 2006; 130: 1878-1881.

26. Warner, T.F., Louie, R., Hafez, G.R., Chandler, E.:

\section{Comentario al trabajo Tumor maligno de la vaina del nervio periférico (MPNST) glandular de la órbita de A. Romero Rojas y cols.}

Los autores describen un caso de presentación muy inhabitual, un paciente en edad infantil afecto de neurofibromatosis tipo 1, que ha sido diagnosticado y tratado por
Malignant nerve sheath tumor containing endocrine cells. Am J Surg Pathol. 1983; 7: 583-590.

27. Weiss, S.W.: Malignant tumors of peripheral nerves. In: Enzinger FM, Weiss SW, editors. Soft tissue tumors. St. Louis: C.V. Mosby; 2008. p 903-941.

28. Wong, S.Y., Ming, T., Tan, Y.O., Best, P.V.: Malignant glandular triton tumor. Cancer. 1991; 67: 1076-1083.

29. Woodruff, J.M., Chernik, N.L., Smith, M.C., Millett, W.B., Foote, F.W.: Peripheral nerve tumors with rhabdomyosarcomatous differentiation (Malignant "triton" tumors). Cancer. 1973; 32: 426-439.

30. Woodruff, J.M.: Peripheral nerve tumors showing glandular differentiation (glandular schwannomas). Cancer. 1976; 37: 2399-2413.

31. Woodruff, J.M., Christensen, W.N.: Glandular peripheral nerve sheath tumors. Cancer. 1993; 72 :3618-3628.

32. Woodruff, J.M.: Pathology of tumors of the peripheral nerve sheath in type 1 neurofibromatosis. Am J Med Genet 1999; 89: 23-30.

33. Woodruff, J.M., Kourea, H.P., Louis, D.N., Scheithauer, BW.: Malignant peripheral nerve sheath tumor (MPNST). In: Kleihues P, Cavenee WK, eds. Pathology and Genetics of Tumours of the Nervous System. Lyon, France: IARC Press; 2000: 172-174. World Health Organization Classification of Tumours.

34. Zhou, H., Coffin, C.M., Perkins, S.L., Tripp, S.R., Liew, M., Viskochil, D.H.: Malignant peripheral nerve sheath tumor: a comparison of grade, immunophenotype, and cell cycle/growth activation marker expression in sporadic and neurofibromatosis 1-related lesions. Am J Surg Pathol. 2003; 27:1337-1345.

Romero-Rojas, A.E.; Díaz-PérezJ.A.; Lozano-Castillo, A.: Tumor maligno de la vaina del nervio periférico (MPNST) glandular de la órbita: primera descripción de la literatura de localización orbitaria en un paciente con neurofibromatosis tipo 1. Neurocirugía 2010; 21:37-45.

Correspondencia: Dr. Alfredo Ernesto Romero Rojas, Grupo de Patología, Instituto Nacional de Cancerología, Cra 9 No 1-85, Bogotá Colombia;

e-mail: aromero@cancer.gov.co un tumor maligno periférico de vainas nerviosas (MPNST en el acrónimo de la denominación de la OMS) con diferenciación divergente y componente glandular maligno. 
El paciente expresaba como criterios de la enfermedad de base manchas café con leche en la piel, hiperpigmentación axilar, nódulos iridianos de Lisch, displasia ósea de ala de esfenoides y el tumor con áreas reconocibles de neurofibroma plexiforme. Desconocemos si el paciente había heredado la enfermedad de sus padres y si se ha realizado estudio molecular. El paciente fue estudiado mediante RM diagnosticando un tumor de partes blandas intraorbitario, con afectación dural por la ausencia displásica de pared orbitaria. Se realizó un tratamiento quirúrgico con extirpación tumoral completa incluyendo meninges afectadas, y reconstrucción plástica, no realizándose tratamiento adyuvante. A los 4 meses del tratamiento el paciente se encuentra asintomático.

Efectivamente, la afectación orbitaria por un MPNST es un hecho infrecuente, aunque reconocido ${ }^{1}$, y el hallazgo de componente glandular en la neoplasia es un hecho excepcional, existiendo pocos casos descritos en la literatura ${ }^{5}$, debiendo considerarse su localización en órbita como un hallazgo circunstancial. Una revisión de la literatura con criterios más amplios permite localizar una descripción realizada en idioma ruso $^{3}$, en el que se mencionan aparentemente ocho casos más de neurofibroma con diferenciación glandular, comportamiento maligno y localización orbitaria. El desarrollo de un MPNST es característico de los pacientes afectos de neurofibromatosis tipo 1, diagnosticándose aproximadamente en el $2 \%$ de estos ${ }^{2}$, y siendo pacientes afectos por la enfermedad en el $50 \%$ al menos de los casos con MPNST, tanto como neoplasia de novo o como evolución maligna de un neurofibroma plexiforme previo, de forma espontánea o inducida tras un tratamiento con radioterapia ${ }^{2}$. La edad de presentación de esta neoplasia es infanto-juvenil en los pacientes NF1, y adulto en los casos esporádicos. Los estudios genéticos, paradójicamente, han mostrado afectaciones tanto del cromosoma 17, donde se localiza el gen NF1, como del cromosoma 22, donde se localiza el gen NF2, junto a otras anomalías ${ }^{4,5}$. La diferenciación divergente se observa en focos de la neoplasia hasta en el $50 \%$ de los casos, con formaciones mesenquimales, epiteloides e incluso glandulares, como el descrito, lo que sirve como argumento para aquellos autores que ponen en duda un origen exclusivo de los MPNST en la malignización de una célula de Schwann ${ }^{6}$. La observación de una diferenciación glandular, con características intestinales, y con postividad característica inmunohistoquímica a CEA, citoqueratinas y sustancias neuroendocrinas, paradójicamente se asocia a un peor pronóstico, con una super- vivencia media de 2 años tras tratamiento. El tratamiento recomendado en la literatura, a pesar de este pronóstico, es la extirpación quirúrgica radical, con abordajes y reconstrucciones complejas como en el caso descrito, recomendándose habitualmente un tratamiento adyuvante con radioterapia $^{1,2,5,6}$. La quimioterapia, hasta la actualidad, no ha demostrado utilidad, estando en estudio líneas de tratamiento dirigidas a dianas moleculares.

En el caso descrito llama la atención la asociación de la localización de la neoplasia con una displasia ósea de ala de esfenoides, como ocurre también en otros pacientes NF1 en los que se observa displasia ósea vecina a neurofibroma plexiforme maligno o no, en localización orbitaria y en tibia (observaciones personales). Esta asociación hace pensar en una posible relación causa-efecto de origen congénito de estos fenómenos en algunos pacientes NF1 y, por otra parte, explican la particular complejidad que ofrecen los abordajes quirúrgicos para su resolución.

\section{Bibliografía}

1. Aydin, M.D., Yildirim, U., Gundogdu, C., Dursun, O., Uysal, H.H., Ozdikici, M.: Malignant peripheral nerve sheath tumor of the orbit: case report and literatura review. Skull Base 2004; 14: 109-114.

2. Evans, D.G.R., Baser, M.E., McGaughran, Sharif, S., Howard, E., Moran, A.: Malignant peripheral nerve sheat tumours in neurofibromatosis 1. J Med Genet 2002; 39: 311314.

3. Matsko, D.E., Nikonov, A.A.: Unique tissue differentiation in peripheral nervous system tumors (glandular neurofibroma). Arkh Patol 1981; 43: 41-44.

4. Rey, J.A., Bello, M.J., Kusak, M.E., de Campos, J.M., Pestaña, A.: Involvemernt of 22q12 in a neurofibrosarcoma in neurofibromatosis type 1. Cancer Genet Cytogenet 1993; 66: 28-32.

5. Sheithauer, B.W., Louis, D.N., Hunter, S., Woodruff, J.M., Antonescu, CR.: Malignant peripheral nerve sheat tumour (MPNST). En WHO Classification of Tumours of the Central Nervous System, Louis DN, Ohgaki H, Wiestler OD, Cavenee WK eds. IARC, Lyon 2007. 4th edition. Pg 160-162.

6. Scheithauer, B.W., Woodruff, J.M., Erlandson, R.A.: Primary malignant tumors of peripheral nerve. En Tumours of the Peripheral Nervous System, Atlas of Tumor Pathology. AFIP, Washington DC, 1999, cap 11, 303-369.

J.M. Campos Madrid 